\title{
Adaptação Portuguesa do Questionário de Coparentalidade: Análise Fatorial Confirmatória e Estudos de Validade e Fiabilidade
}

\author{
Portuguese Adaptation of the Coparenting Questionnaire: Confirmatory \\ Factor Analysis, Validity and Reliability
}

\author{
Marta Figueiredo Pedro* \& Maria Teresa Ribeiro \\ Universidade de Lisboa, Lisboa, Portugal
}

\begin{abstract}
Resumo
Este estudo teve como objetivo adaptar e avaliar as propriedades psicométricas, a validade e a fiabilidade da versão portuguesa do Coparenting Questionnaire (Margolin, Gordis, \& John, 2001). O Coparenting Questionnaire é composto por 14 itens organizados em três subescalas: Cooperação, Triangulação e Conflito. O estudo foi realizado com uma amostra de 1133 indivíduos (530 pais e 603 mães), com filhos pré-adolescentes. A validade fatorial foi testada através da análise fatorial confirmatória. Os resultados replicam a estrutura da versão original composta por três fatores: Cooperação, Triangulação e Conflito. Os valores dos índices de ajustamento revelam uma boa qualidade de ajustamento do modelo aos dados, para pais $(\mathrm{CFI}=0,94, \mathrm{RMSEA}=0,06, \mathrm{SRMR}=0,05) \mathrm{e}$ mães $(\mathrm{CFI}=0,96, \mathrm{RMSEA}=0,06, \mathrm{SRMR}=0,05)$. A escala apresenta coeficientes de consistência interna entre o aceitável e o elevado $(0,71<\alpha<0,88)$, bem como valores adequados de validade convergente e discriminante. Os resultados suportam a validade do construto de coparentalidade e sugerem a versão portuguesa do Coparenting Questionnaire como uma medida válida do construto. Palavras-chave: Análise fatorial confirmatória, coparentalidade, cooperação, triangulação, conflito.
\end{abstract}

\begin{abstract}
The purpose of this study was to adapt and examine the psychometric properties, validity and reliability of the Portuguese version of the Coparenting Questionnaire (Margolin, Gordis, \& John, 2001). The Coparenting Questionnaire includes 14 itens organized in 3 subscales: Cooperation, Triangulation and Conflict. Data were collected from 1,133 participants (530 fathers and 603 mothers) with preadolescent children. Factorial validity was tested through confirmatory factor analysis. The results replicated the original three-factor structure: Cooperation, Triangulation and Conflict. Confirmatory factor analysis indicated a good fit of the model for fathers (CFI $=.94$, RMSEA $=.06$, SRMR $=$ $.05)$ and for mothers $(\mathrm{CFI}=.96, \mathrm{RMSEA}=.06, \mathrm{SRMR}=.05)$. The instrument presents Cronbach's alpha coefficients between acceptable and $\operatorname{good}(.71<\alpha<.88)$ as well as adequate values for the convergent and discriminant validity. These results support the validity of the coparenting construct and the Portuguese version of the Coparenting Questionnaire as a valid measure.

Keywords: Confirmatory factor analysis, coparenting, cooperation; triangulation, conflict.
\end{abstract}

A literatura familiar sistêmica tem vindo a revelar um interesse crescente no estudo da coparentalidade - a forma como os elementos do casal se relacionam no seu papel de pais (Feinberg, 2003) - considerando-a um componente central das relações interparentais e da vida familiar em geral (Lamela, Castro, \& Figueiredo, 2010). Neste sentido, a influência da coparentalidade na interacção pais-filhos e no desenvolvimento infantil é um dos dados mais consistentes da investigação na área da família (para uma

* Endereço para correspondência: Faculdade de Psicologia, Universidade de Lisboa, Alameda da Universidade, Lisboa, Portugal 1649-013. E-mail: mmpedro@psicologia.ulisboa.pt e mteresaribeiro@psicologia.ulisboa.pt revisão, ver Feinberg, 2003), salientando-se a importância do estudo aprofundado desse processo. O presente trabalho tem como objetivo validar, para o contexto português, a estrutura fatorial de um dos instrumentos mais utilizados actualmente na avaliação das dimensões da coparentalidade, o Coparenting Questionnaire (CQ; Margolin, Gordis, \& John, 2001).

Embora não haja consenso quanto ao fato da coparentalidade ser um construto conjugal ou parental, as relações coparental e conjugal devem ser entendidas como conceptualmente distintas, apesar de significativamente associadas (Bonds \& Gondoli, 2007; McHale, Lauretti, Talbot, \& Pouquette, 2002). Segundo Margolin et al. (2001), a coparentalidade não é apenas uma dimensão da 
Pedro, M. F. \& Ribeiro, M. T. (2015). Adaptação Portuguesa do Questionário de Coparentalidade: Análise Fatorial Confirmatória e Estudos de Validade e Fiabilidade.

relação conjugal, pois desempenha um papel singular no sistema familiar. Focando-se nos assuntos relativos à educação da criança, a coparentalidade inclui apenas aspectos diretamente relacionados com a parentalidade (McHale et al., 2002). A coparentalidade difere, portanto, da relação conjugal, estando esta centrada no bem-estar dos cônjuges (Caldera \& Lindsey, 2006).

Essa distinção entre as relações coparental e conjugal tem sido apoiada por várias evidências empíricas que indicam que a coparentalidade funciona como um preditor mais próximo das práticas parentais, da relação progenitor-criança (e.g., Bonds \& Gondoli, 2007; Margolin et al., 2001) e de vários aspectos do ajustamento infantil (e.g., Caldera \& Lindsey, 2006), do que a qualidade conjugal em geral. Desse modo, tem sido sugerido por diversos autores que a coparentalidade constitui um alvo de intervenção mais poderoso e eficaz na melhoria da parentalidade, comparativamente à relação conjugal em geral (e.g., Bonds \& Gondoli, 2007). A investigação mostra que melhorar a qualidade da conjugalidade é uma tarefa árdua e que a intervenção focada na relação coparental poderá ter resultados mais robustos na interacção pais-filhos (Feinberg, 2003). A coparentalidade constitui, assim, um importante ponto de intervenção ao nível da melhoria do funcionamento familiar e do desenvolvimento da criança (Feinberg, Brown, \& Kan, 2012).

\section{Conceptualização de Coparentalidade}

Um dos principais desafios no estudo da coparentalidade relaciona-se com a falta de acordo relativamente à sua conceptualização. Têm sido propostos vários modelos de coparentalidade, diferindo no número e definição das dimensões apresentadas (e.g., Feinberg, 2003; Margolin et al., 2001), embora seja consensual que se trata de um constructo multidimensional (Feinberg et al., 2012; Lamela, Costa, \& Figueiredo, 2010).

No presente estudo, debruçamo-nos sobre o modelo proposto por Margolin e colegas (2001). Esse modelo propõe que a coparentalidade é constituída por três dimensões principais. A primeira dimensão corresponde ao conflito entre as figuras parentais relacionado com os assuntos da parentalidade. A quantidade, frequência e severidade dos desacordos ou discussões acerca da educação dos filhos, bem como as tentativas de boicotar ou enfraquecer a parentalidade do cônjuge, caracterizam essa dimensão. A segunda dimensão diz respeito à cooperação entre os pais, refletindo em que medida estes se valorizam, apoiam e respeitam mutuamente no exercício das suas funções parentais. Os autores destacam a cooperação como um componente essencial no desenvolvimento de uma parentalidade eficaz, revelando, por um lado, um sentimento de partilha dos deveres parentais e, por outro, a segurança de que a outra figura parental está disponível para a criança. A terceira dimensão é a triangulação. A triangulação representa uma distorção das fronteiras entre os subsistemas parental e pai/mãe-filho, onde um dos pais estabelece uma coalisão intergeracional com a criança, excluindo ou enfraquecendo a outra figura parental (Kerig, 2005). A criança é "arrastada" para o conflito interparental e pressionada a aliar-se a um dos pais contra o outro, podendo servir de confidente e transmitir mensagens entre as figuras parentais (Buchanan, Maccoby \& Dornbusch, 1991). Vários estudos com famílias nucleares e divorciadas mostram que a triangulação se encontra associada ao desajustamento emocional da criança e do adolescente, bem como a dificuldades conjugais e familiares (Buchanan et al., 1991; Kerig, 1995).

\section{O Coparenting Questionnaire}

O Coparenting Questionnaire (CQ; Margolin et al., 2001) foi desenvolvido para avaliar a percepção que os cônjuges têm um do outro enquanto pais. O desenvolvimento do CQ surgiu da necessidade de se criar uma medida que reunisse um conjunto de características que permitissem colmatar algumas limitações de instrumentos já existentes. Em primeiro lugar, o CQ é uma escala que contempla dimensões positivas e negativas da coparentalidade, ao mesmo tempo que avalia dinâmicas de triangulação. Medidas anteriores da relação coparental ou se focavam exclusivamente em aspectos positivos da coparentalidade, como o apoio interparental (Parenting Alliance Inventory; Abidin \& Brunner, 1995), ou, apesar de avaliarem dimensões positivas e negativas da coparentalidade, como o conflito interparental, não avaliavam a triangulação (Coparenting Scale; McHale, 1997). Adicionalmente, o CQ é uma medida direcionada unicamente para a avaliação da coparentalidade, contrariamente a instrumentos anteriores que avaliavam processos coparentais em conjunto com a unidade familiar (Coparenting Scale; McHale, 1997). Por último, uma das dificuldades inerentes às medidas de auto-relato com temáticas da parentalidade é a elevada desejabilidade social (Morsbach \& Prinz, 2006). O CQ diminui esse efeito, uma vez que a coparentalidade é avaliada pelo parceiro e não pelo próprio (ou seja, as mães respondem relativamente ao comportamento coparental dos pais, e os pais respondem relativamente ao comportamento coparental das mães), contrariamente aos instrumentos já existentes.

A versão original do CQ foi validada com recurso a três amostras independentes (uma amostra piloto de mães de crianças em idade escolar, uma amostra de pais com filhos em idade pré-escolar e outra com pais de pré-adolescentes). As dimensões da coparentalidade foram ainda correlacionadas com a percepção da criança do conflito interparental e da triangulação, bem como com observações da comunicação conjugal (Margolin et al., 2001). A versão final da escala ficou constituída por 14 itens, cotados numa escala de tipo Likert variando de (1) Nunca a (5) Sempre. Os autores reportaram níveis satisfatórios de consistência interna nas três sub-escalas (alfas de Cronbach entre 0,69 e 0,87 ) para pais e mães.

A fácil administração e a sua dimensão reduzida, bem como as suas qualidades psicométricas, contribuíram para que o CQ seja um dos instrumentos de auto-relato 
actualmente mais utilizados na avaliação das dinâmicas da coparentalidade (e.g., Baril, Crouter, \& McHale, 2007; Barzel \& Reid, 2011; Kolak \& Volling, 2007; Linares, Rhodes, \& Montalto, 2010). Recentemente, Barzel e Reid (2011) confirmaram a estrutura fatorial do CQ através de uma análise fatorial confirmatória, mas numa amostra muito pequena de pais de diabéticos $(N=61)$, o que pode ter resultado em parâmetros estimados pouco fiáveis. Salienta-se ainda que, até à data, não existe uma versão deste instrumento em língua portuguesa. O presente estudo tem assim como objetivo adaptar a versão portuguesa do $\mathrm{CQ}$, bem como testar a sua estrutura fatorial e analisar a validade convergente e discriminante, numa amostra comunitária (à semelhança da amostra de Margolin et al., 2001) de pais com filhos pré-adolescentes, realizando a primeira adaptação do CQ à população portuguesa.

\section{Método}

\section{Participantes}

A amostra foi constituída por 1133 sujeitos (530 pais e 603 mães) casados $(n=992)$ ou em união de facto $(n$ $=141)$, residentes na zona de Lisboa e Vale do Tejo $(59,2 \%)$ e da zona Litoral Oeste $(38,3 \%)$, com filhos pré-adolescentes de idades compreendidas entre os 9 e os 13 anos $(M=10,97 ; D P=0,92)$. Esta amostra fez parte de uma investigação mais abrangente, acerca das associações existentes entre a relação conjugal e a relação pais-filhos, na qual se examinou o papel da coparentalidade. Os pais que participaram no estudo tinham idades compreendidas entre os 24 e os $69 \operatorname{anos}(M=43,03, D P=6,11)$ e diferentes níveis de escolaridade (até ao $4^{\circ}$ ano de escolaridade $-9,1 \%$; do $5^{\circ}$ ao $12^{\circ}$ ano de escolaridade - 55,2\%; ensino superior $-31 \%)$. As mães tinham idades compreendidas entre os 26 e os 62 anos $(M=40,16, D P=5,29)$ e diferentes níveis de escolaridade (até ao $4^{\circ}$ ano de escolaridade $-7,3 \%$; do $5^{\circ}$ ao $12^{\circ}$ ano de escolaridade $-52,5 \%$; ensino superior $-40,2 \%)$. A maioria dos participantes era de raça branca (98\%) e tinha em média 2 filhos.

\section{Procedimento}

Os participantes foram recrutados de 14 escolas. A aprovação ética e o consentimento informado foram obtidos junto do Conselho Executivo de cada uma das escolas, previamente ao início do estudo. Após recolhidas as respectivas autorizações das escolas para se iniciar o estudo, os pais foram contatados por intermédio dos seus filhos, através da distribuição de cartas aos alunos do $5^{\circ}$ ano e do $6^{\circ}$ ano, descrevendo o estudo e convidando as famílias a participarem. Todos os pais entregaram o consentimento informado de que iriam participar num estudo sobre a associação entre a relação conjugal e as interações pais-filhos. Os questionários dos pais foram enviados para casa através dos filhos, num envelope selado. Cada envelope continha dois conjuntos de questionários (um para o pai e outro para a mãe), com instruções para os progenitores responderem em separado e de forma independente (Anexo). De modo a clarificar eventuais dúvidas que pudessem surgir por parte dos pais, foi fornecido o contato do investigador principal. Os questionários eram devolvidos através de um envelope pré-pago fornecido a cada família, ou entregues ao professor da criança, se os pais assim preferissem. Os questionários eram anónimos e os participantes eram assegurados de que os dados recolhidos iriam ser utilizados apenas para fins de investigação.

\section{Instrumentos}

O Coparenting Questionnaire (CQ; Margolin et al., 2001) é um questionário de hetero-relato construído para avaliar as percepções que os cônjuges/parceiros têm um do outro enquanto pais. As respostas avaliam a percepção do próprio (mãe ou pai) acerca do comportamento do parceiro na relação coparental. O CQ é constituído por três subescalas distintas - cooperação, conflito e triangulação. Resultados elevados indicam níveis elevados de cooperação, conflito e triangulação. A subescala Cooperação inclui cinco itens (itens 1 a 5) relativos ao nível com que os elementos do casal se apoiam e respeitam enquanto pais. A subescala Conflito (itens 10 a 14) diz respeito ao conflito interparental acerca dos assuntos que dizem respeito à educação dos filhos e também inclui cinco itens. $\mathrm{O}$ item 13 é invertido. Por último, a subescala Triangulação é composta por quatro itens (itens 6 a 9) que refletem as tentativas dos pais para estabelecerem uma coalisão com a criança que exclua o parceiro coparental ou ponha em causa a sua autoridade parental. A versão portuguesa do CQ (Questionário da Coparentalidade [QC]; Anexo) foi obtida através de um procedimento de retroversão, realizado por indivíduos bilingues.

Adicionalmente ao preenchimento da versão portuguesa do CQ, os participantes responderam ainda a um questionário de satisfação conjugal (Escala de Avaliação da Satisfação em Áreas da Vida Conjugal [EASAVIC]; Narciso \& Costa, 1996) e outro de práticas parentais (EMBU-Pais [EMBU-P]; Canavarro \& Pereira, 2007). A EASAVIC avalia a satisfação conjugal em várias áreas da vida conjugal e é um instrumento de auto-relato composto por duas escalas - Amor e Funcionamento - constituído por 44 itens, avaliados numa escala de Likert de (1) Não satisfeito a (6) Completamente satisfeito. A EASAVIC apresenta um bom nível de consistência interna para pais $(\alpha$ Amor $=0,98, \alpha$ Funcionamento $=0,93)$ e para mães $(\alpha$ Amor $=0,98, \alpha$ Funcionamento $=0,94)$. Resultados elevados refletem níveis elevados de satisfação conjugal. O EMPU-P é a versão portuguesa do EMBU-Pais (Castro, Pablo, Gómez, Arrindell, \& Toro, 1997), a versão para pais da escala sueca Egna Minnen av Barndoms Uppfostram (Memórias de Infância; Perris, Jacobsson, Lindström, von Knorring, \& Perris, 1980), e avalia a percepção que os progenitores têm dos seus estilos parentais relativamente aos filhos. O EMBU-P é uma medida de auto-relato com 42 itens, composta por três susbescalas: Suporte Emocional, 
Pedro, M. F. \& Ribeiro, M. T. (2015). Adaptação Portuguesa do Questionário de Coparentalidade: Análise Fatorial Confirmatória e Estudos de Validade e Fiabilidade.

Rejeição e Tentativa de Controlo. Os itens são avaliados numa escala de Likert de (1) Não, nunca a (4) Sim, sempre. A subescala Suporte Emocional é constituída por 14 itens que manifestam a expressão verbal e física de apoio afectivo, aceitação e disponibilidade física e psicológica por parte dos pais. A subescala Rejeição inclui 17 itens referentes a hostilidade/agressão verbal e física e à não aceitação da criança. A subescala Tentativa de Controlo é composta por 11 itens que traduzem intenções ou acções parentais com o objectivo de gerir o comportamento da criança. A consistência interna para as três subescalas é aceitável: Suporte Emocional (mães $\alpha=0,79$, pais $\alpha=$ 0,87 ), Rejeição (mães $\alpha=0,77$, pais $\alpha=0,78$ ) e Controlo (mães $\alpha=0,66$, pais $\alpha=0,73$ ). Os dados sócio-demográficos foram recolhidos através de um questionário construído para o efeito.

\section{Análise Estatística}

Numa primeira fase, foi realizada a análise descritiva dos dados (médias e desvios-padrão) através do software estatístico SPSS Statistics 19. Em seguida, avaliou-se a validade fatorial do CQ na amostra portuguesa com uma análise fatorial confirmatória (AFC), utilizando o software Amos 19 (Arbuckle, 2010). Em primeiro lugar, estimou-se o modelo hierárquico original proposto pelos autores (com 3 fatores e um fator de $2^{\mathrm{a}}$ ordem), em separado para pais e mães (Figura 1-Modelo 1). Em segundo lugar, comparou-se o modelo hierárquico com modelos alternativos (Figura 2 ), com base no teste da diferença dos $\chi^{2}$ de modelos aninhados, novamente em separado para pais e mães. O método de Estimation Maximization do SPSS foi utilizado para o tratamento dos dados omissos. A percentagem de dados omissos variou entre $1,5 \%$ e $13,5 \%$.
Para avaliar o ajustamento dos modelos aos dados, consideraram-se os seguintes índices de qualidade de ajustamento: o qui-quadrado $\left(\chi^{2}\right)$, o comparative fit index (CFI), o root-mean-square error of approximation (RMSEA), e o standardized root-mean-square residual (SRMR). De acordo com Hu e Bentler (1999), valores de CFI > 0,95, de RMSEA $<0,06$ e de SRMR $<0,08$, são indicativos de um bom ajustamento do modelo aos dados.

De forma a recolher mais informações sobre a versão portuguesa do CQ, examinou-se ainda a validade convergente e discriminante do instrumento. Para este efeito, as notas do CQ foram correlacionadas com o EMBU e a EASAVIC, para pais e mães em separado.

\section{Resultados}

A Tabela 1 apresenta as médias, os desvio-padrão e as correlações inter-item do CQ. A análise descritiva dos resultados revelou que os pais e as mães recorreram às cinco possibilidades de resposta existentes para cada um dos itens, com as médias de resposta apresentando valores compreendidos entre 1,18 e 4,26, para os itens 8 e 5, respectivamente. Foram ainda observadas correlações variando entre moderadas e altas entre os itens $(0,21 \leq r$ $\leq 0,77)$, corroborando a ideia de que existem variáveis latentes entre os itens. O cálculo do alfa de Cronbach para cada uma das subescalas revelou uma consistência interna elevada e aceitável (para pais $-\alpha$ cooperação $=$ $0,83, \alpha$ triangulação $=0,77$ e $\alpha$ conflito $=0,71 ;$ para mães $-\alpha$ cooperação $=0,88, \alpha$ triangulação $=0,85$ e $\alpha$ conflito $=0,76)$, respeitando o critério sugerido por Nunnally (1978). Os níveis de consistência interna obtidos na versão portuguesa do CQ são assim semelhantes aos obtidos por Margolin et al. (2001).

Tabela 1

Médias, Desvios-Padrão e Correlações Inter-Itens da Versão Portuguesa do CQ $(N=1133)$ para Pais e Mães

\begin{tabular}{lcccccccccccccc}
\hline Items & 1 & 2 & 3 & 4 & 5 & 6 & 7 & 8 & 9 & 10 & 11 & 12 & 13 & 14 \\
\hline 1. Item 1 & $\mathbf{0 , 3 8}$ & 0,69 & 0,50 & 0,55 & 0,45 & $-0,16$ & $-0,29$ & $-0,31$ & $-0,39$ & $-0,27$ & $-0,21$ & $-0,17$ & $-0,28$ & $-0,21$ \\
2. Item 2 & 0,75 & $\mathbf{0 , 3 9}$ & 0,51 & 0,54 & 0,41 & $-0,13$ & $-0,22$ & $-0,28$ & $-0,31$ & $-0,13$ & $-0,16$ & $-0,19$ & $-0,22$ & $-0,14$ \\
3. Item 3 & 0,56 & 0,55 & $\mathbf{0 , 4 0}$ & 0,56 & 0,32 & $-0,31$ & $-0,34$ & $-0,36$ & $-0,36$ & $-0,23$ & $-0,25$ & $-0,27$ & $-0,33$ & $-0,26$ \\
4. Item 4 & 0,57 & 0,56 & 0,63 & $\mathbf{0 , 3 9}$ & 0,39 & $-0,23$ & $-0,29$ & $-0,28$ & $-0,36$ & $-0,25$ & $-0,28$ & $-0,24$ & $-0,28$ & $-0,26$ \\
5. Item 5 & 0,60 & 0,57 & 0,55 & 0,57 & $\mathbf{0 , 2 0}$ & $-0,12$ & $-0,22$ & $-0,19$ & $-0,16$ & $-0,19$ & $-0,19$ & $-0,17$ & $-0,24$ & $-0,18$ \\
6. Item 6 & $-0,36$ & $-0,37$ & $-0,40$ & $-0,38$ & $-0,40$ & $\mathbf{0 , 3 0}$ & 0,53 & 0,40 & 0,37 & 0,24 & 0,31 & 0,44 & 0,19 & 0,45 \\
7. Item 7 & $-0,35$ & $-0,34$ & $-0,37$ & $-0,30$ & $-0,40$ & 0,59 & $\mathbf{0 , 3 9}$ & 0,69 & 0,53 & 0,29 & 0,28 & 0,36 & 0,20 & 0,42 \\
8. Item 8 & $-0,36$ & $-0,36$ & $-0,40$ & $-0,31$ & $-0,41$ & 0,57 & 0,80 & $\mathbf{0 , 3 8}$ & 0,51 & 0,26 & 0,25 & 0,28 & 0,20 & 0,37
\end{tabular}


Psicologia: Reflexão e Crítica, 28(1), 116-125.

\begin{tabular}{|c|c|c|c|c|c|c|c|c|c|c|c|c|c|c|}
\hline 9. Item 9 & $-0,31$ & $-0,30$ & $-0,34$ & $-0,29$ & $-0,39$ & 0,44 & 0,67 & 0,65 & $\mathbf{0 , 3 0}$ & 0,30 & 0,27 & 0,32 & 0,20 & 0,30 \\
\hline 10. Item 10 & $-0,33$ & $-0,28$ & $-0,30$ & $-0,30$ & $-0,37$ & 0,41 & 0,42 & 0,41 & 0,39 & 0,49 & 0,59 & 0,34 & 0,18 & 0,40 \\
\hline 11. Item 11 & $-0,33$ & $-0,31$ & $-0,30$ & $-0,36$ & $-0,37$ & 0,39 & 0,39 & 0,38 & 0,38 & 0,62 & $\mathbf{0 , 4 8}$ & 0,41 & 0,16 & 0,35 \\
\hline 12. Item 12 & $-0,25$ & $-0,22$ & $-0,32$ & $-0,27$ & $-0,25$ & 0,45 & 0,39 & 0,39 & 0,33 & 0,32 & 0,41 & 0,40 & 0,25 & 0,41 \\
\hline 13. Item 13 & $-0,33$ & $-0,35$ & $-0,40$ & $-0,38$ & $-0,37$ & 0,37 & 0,32 & 0,34 & 0,26 & 0,28 & 0,34 & 0,39 & 0,33 & 0,19 \\
\hline 14. Item 14 & $-0,34$ & $-0,29$ & $-0,34$ & $-0,34$ & $-0,37$ & 0,55 & 0,51 & 0,55 & 0,44 & 0,39 & 0,38 & 0,44 & 0,40 & 0,35 \\
\hline Média mães & 3,82 & 3,70 & 4,02 & 3,56 & 4,09 & 1,46 & 1,17 & 1,20 & 1,24 & 1,98 & 2,26 & 1,86 & 2,02 & 1,44 \\
\hline $\begin{array}{l}\text { Desvio- } \\
\text { padrão mães }\end{array}$ & 1,11 & 1,19 & 0,94 & 1,21 & 1,11 & 0,79 & 0,58 & 0,59 & 0,62 & 1,09 & 0,99 & 0,84 & 0,84 & 0,77 \\
\hline Média pais & 4,31 & 4,19 & 4,14 & 3,96 & 4,45 & 1,42 & 1,14 & 1,17 & 1,20 & 1,84 & 2,15 & 1,80 & 2,11 & 1,43 \\
\hline $\begin{array}{l}\text { Desvio- } \\
\text { padrão pais }\end{array}$ & 0,76 & 0,90 & 0,86 & 0,99 & 0,82 & 0,77 & 0,48 & 0,51 & 0,54 & 0,97 & 0,97 & 0,82 & 0,86 & 0,80 \\
\hline
\end{tabular}

Nota. As correlações relativas às mães estão indicadas abaixo da diagonal; as correlações relativas aos pais estão indicadas acima da diagonal; as correlações entre pais e mães estão indicadas em negrito na diagonal.

Todas as correlações são significativas ao nível de $p<0,01$.

Os resultados da análise fatorial confirmatória são apresentados na Tabela 2. Os índices de ajustamento revelam que o modelo hierárquico proposto (Figura 1) é adequado aos dados, para pais e mães. De referir que quanto menor o qui-quadrado do modelo, melhor o seu ajustamento. Todos os itens apresentam um peso fatorial superior a 0,40 .

Tabela 2

Estatísticas de Adequação dos Modelos

\begin{tabular}{|c|c|c|c|c|}
\hline Modelos & $\chi^{2}(g l)$ & CFI & RMSEA & SRMR \\
\hline \multicolumn{5}{|l|}{ Mães } \\
\hline Modelo 1 - Hierárquico & $424,10(74)^{*}$ & 0,92 & 0,09 & 0,06 \\
\hline Modelo $1 \mathrm{~b}$ - Hierárquico constrangido & $941,70(76)^{*}$ & 0,80 & 0,14 & 0,29 \\
\hline Modelo $2-3$ Fatores independentes & $941,70(77)^{*}$ & 0,80 & 0,14 & 0,29 \\
\hline Modelo 3 - Unifatorial & $1360,30(77)^{*}$ & 0,70 & 0,17 & 0,10 \\
\hline Modelo 4 - 3 Fatores correlacionados & $424,10(74)^{*}$ & 0,92 & 0,09 & 0,06 \\
\hline Modelo 4 - melhorado & $245,13(72)^{*}$ & 0,96 & 0,06 & 0,05 \\
\hline \multicolumn{5}{|l|}{ Pais } \\
\hline Modelo 1 - Hierárquico & $349,95(74)^{*}$ & 0,90 & 0,08 & 0,07 \\
\hline Modelo $1 \mathrm{~b}$ - Hierárquico constrangido & $614,84(76)$ & 0,80 & 0,12 & 0,21 \\
\hline Modelo 2 - 3 Fatores independentes & $614,84(77)^{*}$ & 0,80 & 0,11 & 0,21 \\
\hline Modelo 3 - Unifatorial & $981,39(77)^{*}$ & 0,66 & 0,15 & 0,10 \\
\hline Modelo 4 - 3 Fatores correlacionados & $349,95(74)^{*}$ & 0,90 & 0,08 & 0,07 \\
\hline Modelo 4 - melhorado & $225,13(71)^{*}$ & 0,94 & 0,06 & 0,05 \\
\hline
\end{tabular}

${ }^{*} p<0,001$. 
Pedro, M. F. \& Ribeiro, M. T. (2015). Adaptação Portuguesa do Questionário de Coparentalidade: Análise Fatorial Confirmatória e Estudos de Validade e Fiabilidade.

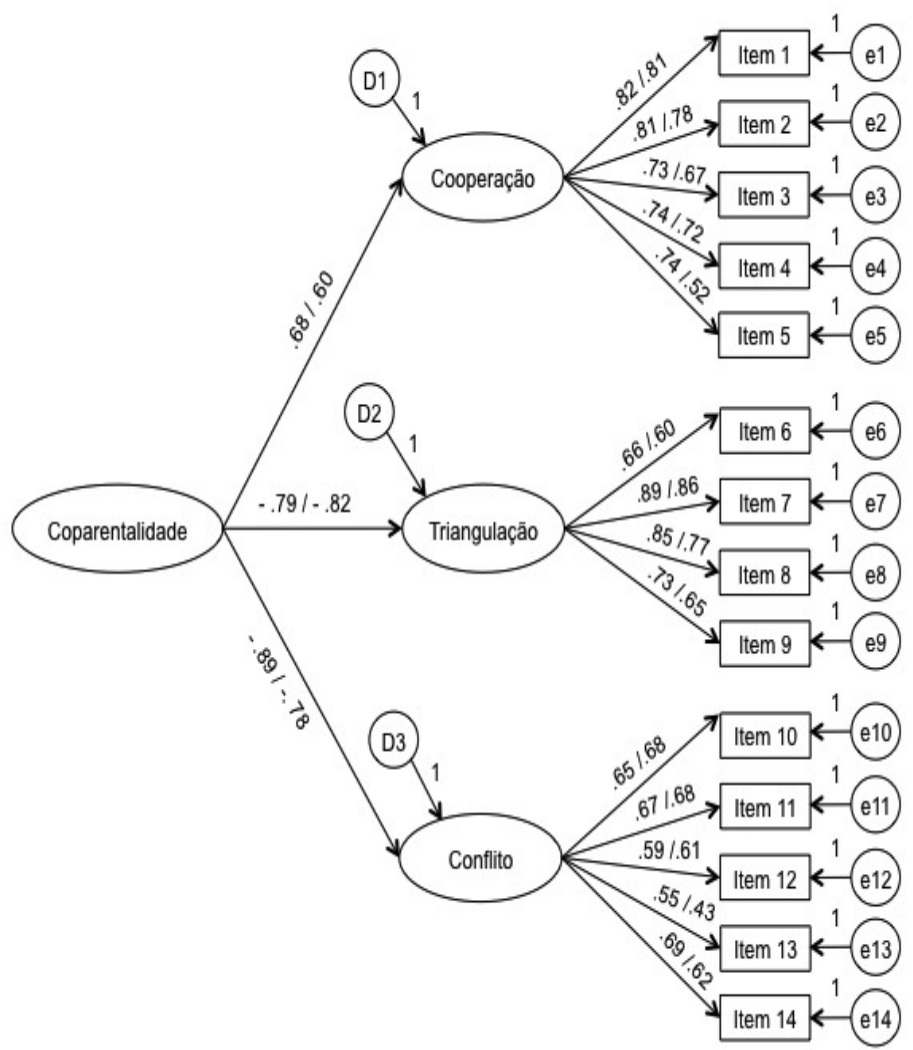

Figura 1. Modelo 1 - Modelo hierárquico como proposto por Margolin et al. (2001) para pais e mães. Os primeiros coeficientes são referentes às mães e os segundos coeficientes são referentes aos pais.

Em seguida, comparou-se o modelo hierárquico (modelo1) com três modelos alternativos (Figura 2): um modelo de três fatores independentes, constituído pelos três subfatores não correlacionados (modelo 2), um modelo unifatorial, no qual todos os itens saturam num único fator de coparentalidade geral (modelo 3) e um modelo de três fatores correlacionados (modelo 4). A análise dos índices de ajustamento (Tabela 2), em conjunto com o teste do $\chi^{2}$, revelou que, para pais e mães, o modelo 1 apresenta um ajustamento significativamente melhor que o modelo 2 [para pais: $\Delta \chi^{2}(3)=264,89, p<0,001$; para mães: $\Delta \chi^{2}(3)$ $=517,60, p<0,001]$ e que o modelo 3 [para pais: $\Delta \chi^{2}(3)=$ $936,20, p<0,001$; para mães: $\left.\Delta \chi^{2}(3)=936,20, p<0,001\right]$. Considerando que do ponto de vista teórico é importante analisar cada uma das dimensões de coparentalidade, comparou-se ainda o modelo $1 \mathrm{com}$ um modelo alternativo de três fatores correlacionados (modelo 4; Figura 2). Dado que os modelos 1 e 4 são estatisticamente iguais e, portanto, não comparáveis, para se poder efetuar a comparação entre os dois modelos constrangeram-se à igualdade todos os pesos fatoriais do modelo 1 (modelo $1 \mathrm{~b}$ ), comparando-se este modelo com o modelo 4 . A análise dos índices de ajustamento (Tabela 2), em conjunto com o teste do $\chi^{2}$, indicou que o modelo 4 revela um ajustamento significativamente melhor que o modelo $1 \mathrm{~b}$, para pais $\left[\Delta \chi^{2}(2)=264,89, p<\right.$ $0,001]$, e mães $\left[\Delta \chi^{2}(2)=517,80, p<0,001\right]$.

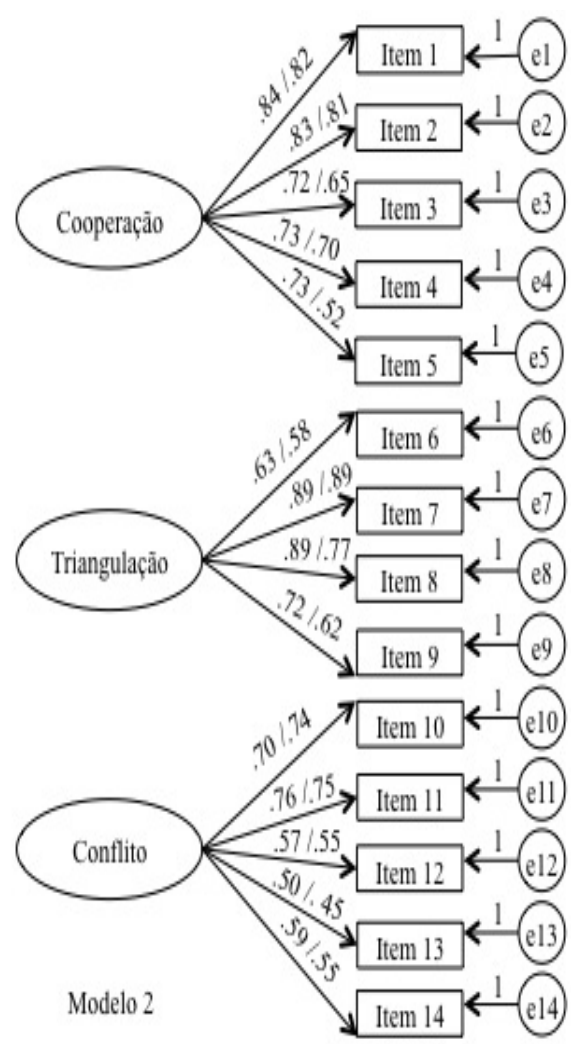



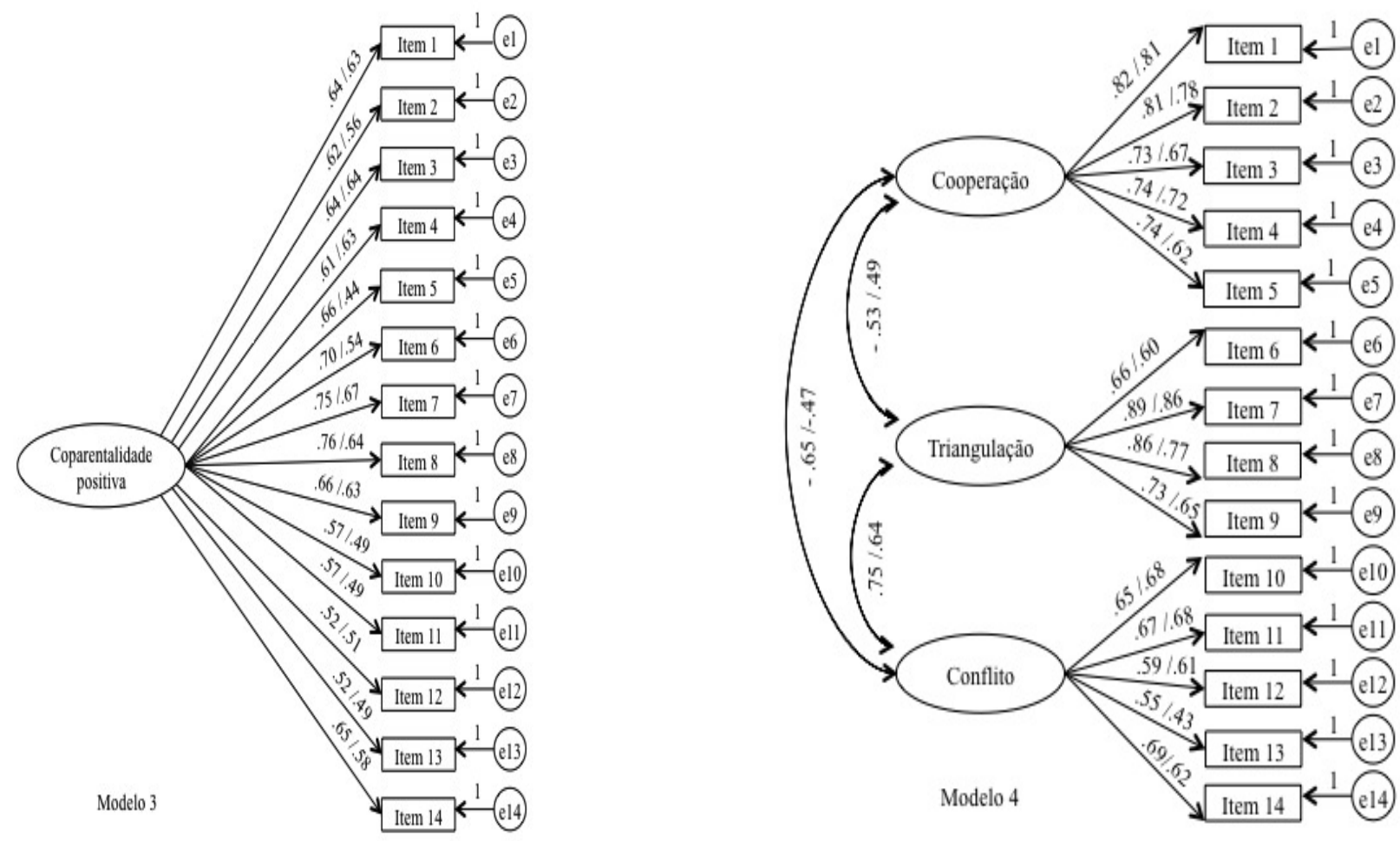

Figura 2. Modelos alternativos. Modelo 2 - Modelo de três fatores independentes; Modelo 3 - Modelo unifatorial; Modelo 4 - Modelo de três fatores correlacionados. Os primeiros coeficientes são referentes às mães e os segundos coeficientes são referentes aos pais.

Posteriormente, e a título exploratório, procedeu-se à melhoria do modelo de três fatores correlacionados (modelo 4), com base na análise dos índices de modificação (IM) calculados pelo Amos (Arbuckle, 2010). De acordo com as sugestões de Maroco (2010), para evitar a capitalização de erros de tipo 1, frequentes aquando da utilização dos IM, procedeu-se apenas à correlação de resíduos nos quais IM sugeria um correlação significativa $\operatorname{com} p<0,001$, tendo-se observado uma diferença entre pais e mães. Nomeadamente, os valores dos IM sugeriram correlações entre os resíduos dos itens 1 e 2, e dos itens 10 e 11, para os modelos dos pais e das mães. Estas modificações são teoricamente justificáveis, uma vez que os resíduos correlacionados pertencem ao mesmo fator. Contudo, para o modelo dos pais, os valores dos IM sugeriram ainda correlações entre os resíduos dos itens 6 e 12 . Uma vez que os itens 6 e 12 se referem ambos a situações de negatividade entre as figuras parentais, a correlação entre os seus resíduos é igualmente justificável teoricamente. Comparativamente com o modelo de três fatores correlacionados inicial, o modelo melhorado apresenta melhores valores de ajustamento (Tabela 2), tanto para pais como para mães.

Na Tabela 3 são apresentados os resultados das correlações entre o CQ, a EASAVIC e o EMBU, separadamente para pais e mães. Conforme o esperado, apesar das medidas se encontrarem significativamente associadas, as correlações são moderadas. Estes resultados indicam que o QC tem validade convergente com a EASAVIC, embora as correlações não sejam demasiado elevadas que sugiram sobreposição entre os construtos. Verifica-se igualmente a presença de validade convergente entre o QC e o EMBU, exceto para a dimensão Controlo. As correlações relativas à dimensão Controlo indicam a presença de validade discriminante entre o Controlo e o QC.

\section{Discussão}

O presente estudo teve como objetivo testar a validade fatorial da versão portuguesa do CQ, numa amostra portuguesa de pais e mães com filhos pré-adolescentes. Para tal, realizou-se uma análise fatorial confirmatória com modelos de equações estruturais, de forma a testar a estrutura hierárquica tri-fatorial com um fator de $2^{\mathrm{a}}$ ordem, proposta originalmente por Margolin et al. (2001). Os resultados obtidos permitem concluir que a versão portuguesa do CQ apresenta qualidades psicométricas aceitáveis ao nível da sua validade fatorial e fiabilidade. A estrutura tri-fatorial original manteve-se na nossa amostra, à semelhança do estudo de Barzel e Reid (2011), salientando a relevância da cooperação entre as figuras parentais, da triangulação da criança e do conflito interparental como dimensões essenciais da coparentalidade. Contudo, contrariamente ao obtido por Margolin et al. (2001), a estrutura de três fatores correlacionados (e não a estrutura hierárquica) apresentou um melhor ajustamento aos dados. Neste sentido, importa referir que foram observadas correlações elevadas entre as escalas da versão portuguesa do CQ, nomeadamente, entre as dimensões Triangulação e Conflito $(r=0,71)$. À 
Pedro, M. F. \& Ribeiro, M. T. (2015). Adaptação Portuguesa do Questionário de Coparentalidade: Análise Fatorial Confirmatória e Estudos de Validade e Fiabilidade.

Tabela 3

Correlações entre as Pontuações do CQ e as Pontuações do EMBU-P e EASAVIC

\begin{tabular}{lcccccc}
\hline & \multicolumn{3}{c}{ Mães } & \multicolumn{3}{c}{ Pais } \\
\cline { 2 - 7 } & Cooperação & Conflito & Triangulação & Cooperação & Conflito & Triangulação \\
\hline EASAVIC & & & & & & \\
Amor & $0,48^{* *}$ & $-0,37^{* *}$ & $-0,41^{* *}$ & $0,44^{* *}$ & $-0,33^{* *}$ & $-0,33^{* *}$ \\
Funcionamento & $0,44^{* *}$ & $-0,37^{* *}$ & $-0,35^{* *}$ & $0,39^{* *}$ & $-0,34^{* *}$ & $-0,27^{* *}$ \\
EMBU-P & & & & & & \\
Suporte Emocional & $0,28^{* *}$ & $-0,27^{* *}$ & $-0,11^{*}$ & $0,52^{* *}$ & $-0,34^{* *}$ & $-0,24^{* *}$ \\
Controlo & $0,11^{*}$ & $-0,04$ & 0,01 & $0,24^{* *}$ & 0,04 & 0,03 \\
Rejeição & $-0,12^{*}$ & $0,22^{* *}$ & $0,20^{*}$ & $-0,12^{*}$ & $0,35^{* *}$ & $0,28^{* *}$ \\
\hline
\end{tabular}

Nota. EMBU-P = versão portuguesa para pais da escala Memórias de Infância; EASAVIC = Escala de Avaliação da Satisfação em Áreas da Vida Conjugal.

$* p<0,05 ; * *<<0,01$.

partida, estas correlações elevadas poderiam pôr em causa a utilidade das escalas na prática e questionar se as mesmas têm capacidade para dar um contributo independente na previsão de outras variáveis importantes. Contudo, estes dados são consistentes com os resultados de estudos anteriores que também observaram correlações elevadas entre as dimensões do CQ (e.g., Barzel \& Reid, 2011; Kolak \& Volling, 2007; Pedro, Ribeiro, \& Shelton, 2012) e que, no entanto, optaram por avaliar as dimensões em separado por estas representarem construtos teóricos distintos. De facto, vários autores defendem a multidimensionalidade do construto de coparentalidade (e.g., Feinberg, 2003; Margolin et al., 2001), e estudos recentes comprovam o impacto distinto da triangulação e do conflito ao nível do bem-estar e de comportamentos de externalização e internalização em crianças e adolescentes (Baril et al., 2007; Buehler \& Welsh, 2009; Caldera \& Lindsey, 2006). Deste modo, o presente estudo reforça assim a ideia de que o constructo de coparentalidade tem uma natureza multidimensional, devendo por isso ser avaliado ao nível dos seus diferentes componentes, embora considerando a correlação entre os mesmos.

Os resultados indicam ainda que a coparentalidade, apesar de significativamente associada à satisfação conjugal e às práticas parentais, constitui um construto distinto que desempenha um papel único no sistema familiar, como aliás tem sido sugerido por vários autores (e.g., McHale et al., 2002). Os dados estão assim de acordo com estudos anteriores que sugerem que a coparentalidade é um preditor mais próximo do comportamento parental, comparativamente à qualidade conjugal, atuando como um mecanismo de mediação entre o conflito conjugal e a parentalidade (Bonds \& Gondoli, 2007; Margolin et al., 2001). Assim, o Questionário de Coparentalidade poderá constituir um instrumento relevante ao nível da intervenção com famílias nas quais o conflito ou dificuldades conjugais influencia as práticas parentais, ajudando a identificar que aspectos da relação parental deverão ser trabalhados de forma a melhorar a qualidade da coparentalidade e, consequentemente, das práticas parentais.

Por outro lado, os resultados do presente estudo evidenciam a adequação da estrutura proposta para os pais e para as mães da amostra portuguesa. Este resultado sugere a generalização do constructo tri-fatorial da coparentalidade a pais e mães portugueses, evidenciando a representação idêntica da coparentalidade em ambos os progenitores. A demonstração deste tipo de generalização é um dado importante, quer no contexto de investigação, quer na prática clínica com famílias, uma vez que permite a comparação entre pais e mães relativamente ao seu comportamento coparental. Ao nível da intervenção parental, esta comparação possibilita não só a identificação de diferenças entre os contributos que ambos os progenitores dão para a relação coparental, mas também o reconhecimento das dimensões da coparentalidade que necessitam de ser trabalhadas, de forma distinta, com o pai e com a mãe. Neste sentido, importa referir o facto de se terem constatado diferenças ao nível dos valores dos MIs, entre pais e mães. Nomeadamente, para o modelo dos pais (mas não para o modelo das mães), os valores dos MIs sugeriram a correlação entre os resíduos do item 6 (“... diz coisas cruéis acerca de mim, ou que me magoam, em frente do nosso filho") e do item 12 ("... discute comigo por causa do nosso filho"). Este resultado poderá sugerir que situações de negatividade coparental que impliquem a triangulação dos filhos através de hostilidade verbal, e situações de conflito interparental relativo à educação dos filhos, poderão ser percebidos de forma mais global e relacionada pelos pais, ao passo que, para as mães, estes dois tipos de negatividade coparental parecem ser compreendidos como aspectos distintos da coparentalidade. Outra explicação alternativa poderá relacionar-se com o facto das mães parecerem exibir uma maior 
tendência para triangularem os filhos, comparativamente aos pais (Krishnakumar \& Buehler, 2000). Considerando que os pais reportam acerca do comportamento das mães, a probabilidade de comportamentos de conflito coparental se relacionarem com comportamentos de triangulação será também maior relativamente ao comportamento materno do que paterno.

O presente estudo apresenta algumas limitações. Em primeiro lugar, utilizou-se uma amostra de conveniência, o que poderá ter influenciado os resultados obtidos, diminuindo o poder de generalização dos resultados. Adicionalmente, foi examinada a validade convergente e discriminante da medida, mas apenas no que toca às variáveis satisfação conjugal e práticas parentais. Por outro lado, a amostra apenas contemplava casais com filhos pré-adolescentes, pelo que a generalização dos resultados a famílias com outras características (e.g., famílias divorciadas, famílias com filhos de outra faixa-etária) deve ser realizada com cautela. Deste modo, sugere-se a realização de mais investigações focadas na validação do $\mathrm{CQ}$, nomeadamente com famílias em fases diferentes do ciclo vital da família (e.g., famílias com filhos em idade pré-escolas, famílias com filhos adolescentes). Importa ainda referir que os questionários eram preenchidos sem a presença dos investigadores, não permitindo assegurar que as questões tenham sido de facto respondidas de forma individual e confidencial. Do mesmo modo, os dois conjuntos de questionários preenchidos pelos pais eram devolvidos num único envelope, aumentando o risco das respostas poderem ser vistas pelo outro elemento do casal e, assim, podendo ter causado constrangimentos na resposta às questões. Por último, deverá ser salientada a questão da não independência dos dados dos progenitores. Apesar dos dados terem sido analisados em separado para pais e mães, respeitando a não-independência das observações e em congruência com estudos anteriores da área da coparentalidade, este procedimento poderá não ter abordado com a complexidade necessária a natureza diádica dos dados. Esta situação constitui um problema na área da Psicologia da Família, pelo que investigações futuras deverão ter em conta este aspecto, realizando análises mais complexas para este tipo de dados diádicos (e.g., análise multinível; Kenny, Kashy, \& Cook, 2006).

Apesar das limitações existentes, este estudo constitui a primeira adaptação do CQ à população portuguesa, proporcionando, assim, um melhor conhecimento das dinâmicas coparentais das famílias portuguesas. A versão portuguesa do CQ apresenta-se como um importante instrumento, quer ao nível da investigação quer na prática clínica com famílias, permitindo avaliar o contributo único de pais e mães ao nível da coparentalidade. Por outro lado, permite a discriminação entre três dimensões distintas da coparentalidade - cooperação, conflito e triangulação - possibilitando a avaliação do impacto único das mesmas em várias áreas do funcionamento familiar e no desenvolvimento da criança e do adolescente.

\section{Referências}

Abidin, R. R., \& Brunner, J. F. (1995). Development of a parenting alliance inventory. Journal of Clinical Child Psychology, 24, 31-40. doi:10.1111/mono.12024

Arbuckle, J. L. (2010). IBM SPSS Amos 19 user's guide. Crawfordville, FL: Amos Development Corporation.

Baril, M. E., Crouter, A. C., \& McHale, S. M. (2007). Processes linking adolescente well-being, marital love, and coparenting. Journal of Family Psychology, 21, 645-654. doi:10.1037/0893-3200.21.4.645

Barzel, M., \& Reid, G. J. (2011). A preliminary examination of the psychometric properties of the Coparenting Questionnaire and the Diabetes-Specific Coparenting Questionnaire in families of children with type I diabetes. Journal of Pediatric Psychology, 36, 606-617. doi:10.1093/jpepsy/jsq103

Bonds, D. D., \& Gondoli, D. M. (2007). Examining the process by which marital adjustment affects maternal warmth: The role of coparenting support as a mediator. Journal of Family Psychology, 21, 288-296. doi:10.1037/0893-3200.21.2.288

Buchanan, C. M., Maccoby, E. E., \& Dornbusch, S. M. (1991). Caught between parents: Adolescents' experience in divorced homes. Child Development, 62, 1008-1029. doi:10.1111/j.1467-8624.1991.tb01586.x

Buehler, C., \& Welsh, D. P. (2009). A process model of adolescent's triangulation into parents' marital conflict: The role of emotional reactivity. Journal of Family Psychology, 23, 167-180. doi:10.1037/a0014976

Caldera, Y. M., \& Lindsey, E. W. (2006). Coparenting, motherinfant interaction, and infant-parent attachment relationships in two-parent families. Journal of Family Psychology, 20, 275-283. doi:10.1037/0893-3200.20.2.275

Canavarro, M. C., \& Pereira, A. I. (2007). A avaliação dos estilos parentais educativos na perspectiva dos pais: A versão portuguesa do EMBU-P. Psicologia: Teoria, Investigacão e Prática, 2, 271-286.

Castro, L., Pablo, J., Gómez, J., Arrindell, W. A., \& Toro, J. (1997). Assessing rearing behaviour from the perspective of the parents: A new form of the EMBU. Social Psychiatry and Psychiatric Epidemiology, 32, 230-235. doi:10.1007/ BF00788243

Feinberg, M. E. (2003). The internal structure and ecological context of coparenting: A framework for research and intervention. Parenting: Science and Practice, 3, 95-131. doi:10.1207/S15327922PAR0302_01

Feinberg, M. E., Brown, L. D., \& Kan, M. L. (2012). A multi-domain self-report measure of coparenting. Parenting: Science and Practice, 12, 1-21. doi:10.1080/15295192.2012.638870

Hu, L., \& Bentler, P. M. (1999). Cutoff criteria for fit indexes in covariance structure analysis: Conventional criteria versus new alternatives. Structural Equation Modeling, 6, 1-55. doi:10.1080/10705519909540118

Kenny, D. A., Kashy, D. A., \& Cook, W. L. (2006). Dyadic data analysis. New York: The Guilford Press.

Kerig, P. K. (1995). Triangles in the family circle: Effects of family structure on marriage, parenting, and child adjustment. Journal of Family Psychology, 9, 28-43. doi:10.1037/08933200.9.1.28

Kerig, P. K. (2005). Revisiting the construct of boundary dissolution: A multidimensional perspective. Journal of Emotional Abuse, 5, 5-42. doi:10.1300/J135v05n02_02

Kolak, A. M., \& Volling, B. L. (2007). Parental expressiveness as a moderator of coparenting and marital relationship quality. Family Relations, 56, 467-478. doi:10.1111/j.17413729.2007.00474.x 
Pedro, M. F. \& Ribeiro, M. T. (2015). Adaptação Portuguesa do Questionário de Coparentalidade: Análise Fatorial Confirmatória e Estudos de Validade e Fiabilidade.

Krishnakumar, A., \& Buehler, C. (2000). Interparental conflict and parenting behaviors: A meta-analytic review. Family Relations, 49, 25-44. doi:10.1111/j.1741-3729.2000.00025.x

Lamela, D., Castro, M., \& Figueiredo, B. (2010). Pais por inteiro: Avaliação preliminar da eficácia de uma intervenção em grupo para pais divorciados. Psicologia: Reflexão e Crítica, 23(2), 334-344.

Lamela, D., Nunes-Costa, R., \& Figueiredo, B. (2010). Modelos teóricos das relações coparentais: Revisão crítica. Psicologia em Estudo, 15(1), 205-216.

Linares, L. O., Rhodes, J., \& Montalto, D. (2010). Perceptions of coparenting in foster care. Family Process, 49, 530-542. doi:10.1111/j.1545-5300.2010.01338.x

Margolin, G., Gordis, E. B., \& John, R. S. (2001). Coparenting: A link between marital conflict and parenting in twoparent families. Journal of Family Psychology, 15, 3-21. doi:10.1037/0893-3200.15.1.3

Maroco, J. (2010). Análise de equações estruturais: Fundamentos teóricos, software \& aplicações. Lisboa, Portugal: ReportNumber.

McHale, J. P. (1997). Overt and covert coparenting processes in the family. Family Process, 36, 183-201. doi:10.1111/j.15455300.1997.00183.x
McHale, J., Lauretti, A., Talbot, J., \& Pouquette, C. (2002). Retrospect and prospect in the psychological study of coparenting and family group process. In J. McHale \& W. Grolnick (Eds.), Retrospect and prospect in the psychological study offamilies. Mahwah, NJ: Lawrence Erlbaum Associates.

Morsbach, S. K., \& Prinz, R. J. (2006). Understanding and improving the validity of self-report of parenting. Clinical Child and Family Psychology Review, 9, 1-21. doi:10.1007/ s10567-006-0001-5

Narciso, I., \& Costa, M. E. (1996). Amores satisfeitos, mas não perfeitos. Cadernos de Consulta Psicológica, 12,115-130.

Nunnally, J. C. (1978). Psychometric theory ( $2^{\text {nd }}$ ed.). New York: McGraw-Hill.

Pedro, M. F., Ribeiro, T., \& Shelton, K. H. (2012). Marital satisfaction and partners' parenting practices: The mediating role of coparenting behavior. Journal of Family Psychology, 26, 509-522. doi:10.1037/a0029121

Perris, C., Jacobsson, L., Lindström, H., von Knorring, L., \& Perris, H. (1980). Development of a new inventory assessing memories of parental rearing behavior. Acta Psychiatrica Scandinavica, 61, 265-274. doi:10.1111/j.1600-0447.1980. tb00581.x

\section{Anexo}

\section{Questionário de Coparentalidade (QC)}

Pense com que frequência o(a) seu/sua cônjuge/companheiro(a)

actua deste modo consigo.

Depois de escolher a sua resposta, assinale-a com um círculo.

$\mathrm{O}$ (a) meu/minha cônjuge/companheiro(a)...

1. ... conta-me muitas coisas acerca do nosso filho.

2. ... põe-me a par de tudo o que acontece durante o dia-a-dia do nosso filho.

3. ... fala muito bem de mim ao nosso filho.

4. ... pergunta a minha opinião sobre assuntos relacionados com o ser pai/ser mãe.

5. ... participa na resolução dos problemas disciplinares relacionados com o nosso filho.

6. ... diz coisas cruéis acerca de mim, ou que me magoam, em frente do nosso filho.

7. ... usa o nosso filho contra mim.

8. ... quando discutimos, tenta manipular o nosso filho para que este tome o partido de um de nós.

9. ... envia-me mensagens pelo nosso filho em vez de falar directamente comigo.

10. ... e eu temos regras diferentes no que diz respeito à alimentação, rotinas diárias, hora de deitar ou trabalhos de casa do nosso filho.

11. ... e eu temos níveis diferentes de exigência relativamente ao comportamento do nosso filho.

12. ... discute comigo por causa do nosso filho.

13. ... concorda com as minhas decisões relativas à disciplina do nosso filho.

14. ... enfraquece, pouco a pouco, a minha posição de pai/mãe.

$\begin{array}{lllll}1 & 2 & 3 & 4 & 5 \\ 1 & 2 & 3 & 4 & 5 \\ 1 & 2 & 3 & 4 & 5 \\ 1 & 2 & 3 & 4 & 5 \\ 1 & 2 & 3 & 4 & 5 \\ 1 & 2 & 3 & 4 & 5 \\ 1 & 2 & 3 & 4 & 5 \\ 1 & 2 & 3 & 4 & 5 \\ & & & & \\ 1 & 2 & 3 & 4 & 5 \\ 1 & 2 & 3 & 4 & 5 \\ & & & & \\ 1 & 2 & 3 & 4 & 5 \\ & & & & \\ 1 & 2 & 3 & 4 & 5 \\ 1 & 2 & 3 & 4 & 5 \\ 1 & 2 & 3 & 4 & 5\end{array}$

